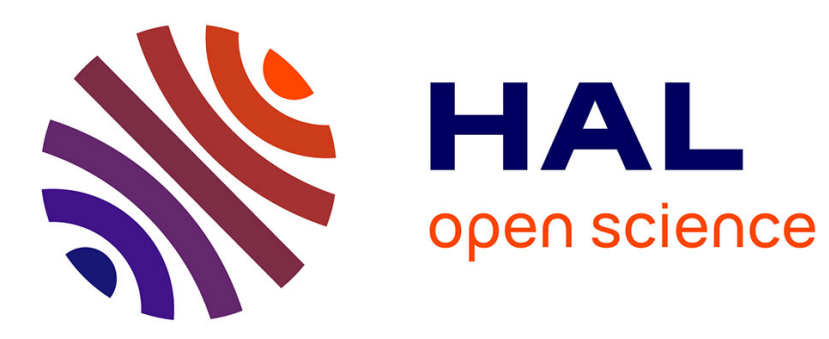

\title{
Linear stability analysis of inclined two-layer stratified flows
}

\author{
M.E. Negretti, S.A. Socolofsky, G.H. Jirka
}

\section{To cite this version:}

M.E. Negretti, S.A. Socolofsky, G.H. Jirka. Linear stability analysis of inclined two-layer stratified flows. Physics of Fluids, 2008, 20 (9), pp.094104. 10.1063/1.2980351 . hal-01022802

\section{HAL Id: hal-01022802}

https://hal-polytechnique.archives-ouvertes.fr/hal-01022802

Submitted on 21 Jul 2014

HAL is a multi-disciplinary open access archive for the deposit and dissemination of scientific research documents, whether they are published or not. The documents may come from teaching and research institutions in France or abroad, or from public or private research centers.
L'archive ouverte pluridisciplinaire HAL, est destinée au dépôt et à la diffusion de documents scientifiques de niveau recherche, publiés ou non, émanant des établissements d'enseignement et de recherche français ou étrangers, des laboratoires publics ou privés. 


\section{AIP | Physics of Fluids}

\section{Linear stability analysis of inclined two-layer stratified flows}

M. Eletta Negretti, Scott A. Socolofsky, and Gerhard H. Jirka

Citation: Physics of Fluids (1994-present) 20, 094104 (2008); doi: 10.1063/1.2980351

View online: http://dx.doi.org/10.1063/1.2980351

View Table of Contents: http://scitation.aip.org/content/aip/journal/pof2/20/9?ver=pdfcov

Published by the AIP Publishing

\section{Articles you may be interested in}

Linear stability of miscible two-fluid flow down an incline

Phys. Fluids 25, 104102 (2013); 10.1063/1.4823855

Linear stability analysis and numerical simulation of miscible two-layer channel flow

Phys. Fluids 21, 042104 (2009); 10.1063/1.3116285

Two-layer stratified flows over pronounced obstacles at low-to-intermediate Froude numbers

Phys. Fluids 21, 044102 (2009); 10.1063/1.3110108

Linear stability of a two-layer film flow down an inclined channel: A second-order weighted residual approach

Phys. Fluids 19, 084106 (2007); 10.1063/1.2757611

Stability of two-layer Newtonian plane Couette flow past a deformable solid layer

Phys. Fluids 16, 4426 (2004); 10.1063/1.1808772

\section{A|P| Journal of}

Journal of Applied Physics is pleased to announce André Anders as its new Editor-in-Chief 


\title{
Linear stability analysis of inclined two-layer stratified flows
}

\author{
M. Eletta Negretti, ${ }^{1, a)}$ Scott A. Socolofsky, ${ }^{2}$ and Gerhard H. Jirka ${ }^{3}$ \\ ${ }^{1}$ LadHyX, École Polytechnique, Palaiseau Cedex 91128, France \\ ${ }^{2}$ Zachry Department of Civil Engineering, Texas A\&M University, College Station, Texas 77843-3136, USA \\ ${ }^{3}$ Institute for Hydromechanics, University of Karlsruhe, D-76131 Karlsruhe, Germany
}

(Received 28 November 2007; accepted 7 August 2008; published online 16 September 2008)

\begin{abstract}
Two-layer stratified flows are commonly observed in geophysical and environmental contexts. At the interface between the two layers, both velocity shear and buoyancy interplay, resulting in various modes of instability. Results from a temporal linear stability analysis of a two-layer stratified exchange flow under the action of a mean advection are presented, investigating the effect of a mild bottom slope on the stability of the interface. The spatial acceleration is directly included in the governing stability equations. The results demonstrate that increasing the bottom slope has a similar effect on the stability of the flow as does increasing the ratio $R$ of the thickness of the velocity mixing layer $\delta_{\nu}$ to that of the density layer $\delta_{\rho}$ as it causes the flow to be more unstable to the Kelvin-Helmholtz instabilities. The transition from Kelvin-Helmholtz modes to stable flow occurs at lower Richardson numbers and wavenumbers compared to the horizontal two-layer flow. KelvinHelmholtz modes are decreasingly amplified for $1<R<\sqrt{2}$. When $\sqrt{2}<R<2$, Kelvin-Helmholtz modes are first amplified and then damped as the Richardson number increases. This suggests that the behavior of the Richardson number alone is not sufficient to predict the stability tendency of the interface. (C) 2008 American Institute of Physics. [DOI: 10.1063/1.2980351]
\end{abstract}

\section{INTRODUCTION}

Two-layer stratified flows are ubiquitous in geophysical sciences such as the exchange of Atlantic Ocean water with the saltier Mediterranean Sea water through the Strait of Gibraltar ${ }^{1}$ or arrested wedge flows typically occurring in fjords or estuaries, when fresh water enters the ocean space. ${ }^{2}$ Even though the density variation is often quite small, the presence of density stratification inhibits vertical motions, thereby influencing nutrient cycling, the distribution of biological and chemical particles, and water quality. In inland water bodies, for instance, the highest concentrations of nutrients are usually found at depths where photosynthesis cannot take place, so that vertical mixing is required to transport these substances into the productive regions. ${ }^{3}$ The combination of external forcing and topographic features, such as underwater slopes, on a two-layer baroclinic flow results in various types of instabilities at the interface. The importance of hydrodynamic instabilities in causing vertical mixing in stratified oceans, in lakes, and in the atmosphere is well recognized. In atmospheric sciences, for example, it has been estimated that instability and mixing cause a significant increase in the drag of atmospheric flows and thus are significant for atmospheric flow modeling and weather forecasting. ${ }^{4}$

The aim of the present linear stability study is to examine the influence of a bottom slope on the stability of the interface in a two-layer stratified flow under the action of a weak mean advection. The bottom slope has the effect of reducing the stabilizing effect of buoyancy at the interface and adds an additional acceleration term which destabilizes

\footnotetext{
${ }^{\text {a) }}$ Telephone: +33(0)1-6933-52-74. Fax: +33(0)1-6933-52-92. Electronic mail: negretti@ladhyx.polytechnique.fr.
}

the flow together with the velocity shear. Comparable studies by means of linear stability analysis investigating the influence of a bottom slope do not exist to date, and therefore the presented results will advance the theoretical knowledge on hydrodynamic instability in two-layer flows.

Earlier studies on hydrodynamic instabilities mostly focused on Kelvin-Helmholtz $(\mathcal{K} \mathcal{H})$ instabilities [see Fig. 1(a)] as they are commonly observed in the atmosphere. ${ }^{5}$ Holmboe $^{6}$ applied temporal linear stability analysis to piecewise linear velocity profiles with a two-layer density structure. With a fixed limit of the Richardson number, he predicted the occurrence of $\mathcal{K H}$ instabilities and reported the possible existence of another type of instability, named after Holmboe $(\mathcal{H})$, when the thickness of the density layer is much smaller than the shear layer [see Fig. 1(b)]. Hazel ${ }^{7}$ conducted detailed numerical experiments and found that $\mathcal{H}$ instabilities can be generated at any shear. He examined the stability of flows where the stability is characterized by the ratio of the shear layer thickness $\delta_{\nu}$ to the density layer thickness $\delta_{\rho}, R=\delta_{\nu} / \delta_{\rho}$, and found critical values for $R$ below which $\mathcal{H}$ instabilities do not exist. Alexakis ${ }^{8}$ studied marginally unstable $\mathcal{H}$ modes and was able to show for a large family of flows and stratification conditions that the modes having a phase speed equal to the maximum or the minimum shear velocity are marginally unstable. This allowed him to determine more precisely the critical value for the transition $\mathcal{K} \mathcal{H}-\mathcal{H}$ of the parameter $R$ to be 2 (see also Ref. 9).

Gelfgat and $\mathrm{Kit}^{10}$ performed a parametric analysis of the temporal and spatial $\mathcal{K} \mathcal{H}$ and $\mathcal{H}$ instabilities and showed that for the same governing parameters the spatial upstream and downstream $\mathcal{H}$ waves have different amplification rates and different absolute phase velocities, with larger differences observed at larger Richardson numbers. Carpenter et al. ${ }^{11}$ 

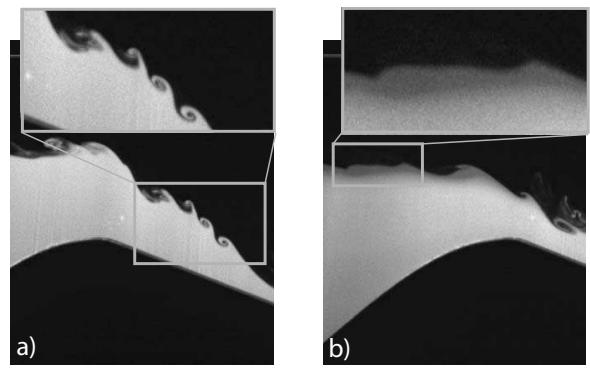

FIG. 1. Interfacial instabilities observed during experiments of two-layer exchange flow over a submerged sill with fluorescent dye diluted in the lower, saltier layer (Ref. 12). (a) $\mathcal{K H}$ instability. (b) $\mathcal{H}$ instability.

examined numerically the evolution and mixing behavior of asymmetric $\mathcal{H}$ instabilities and the results revealed that there are two different mixing mechanisms present, the first being a feature of $\mathcal{K H}$ instabilities and the second was found in asymmetric and symmetric $\mathcal{H}$ instabilities. Pawlak and $\mathrm{Armi}^{13}$ firstly addressed the linear stability analysis from a spatial frame of reference in the case of wedge flow for $R=50$ and $0<J<0.6$. They demonstrated that for arrested wedge flows the spatial theory differs strongly from the temporal theory. The most amplified modes are different and the spatial instability results from a combination of $\mathcal{K H}$ modes and $\mathcal{H}$ instabilities, which was called in their study a hybrid instability region. Later, Ortiz et al. ${ }^{14}$ extended the analysis by Pawlak and $\mathrm{Armi}^{13}$ by analyzing the effect of the mean advection and of changing the Richardson number for piecewise velocity and density profiles. They showed that spatial and temporal linear stability analyses deliver very similar results as long as the mean advection is under a certain threshold $(2 U / \Delta U \leqslant 0.2, U$ being the mean advection and $\Delta U$ the mean velocity difference).

None of the above studies include the presence of the bottom slope in the equations of motion. A similar problem formulation has been addressed by $\mathrm{Smyth}^{15}$ to study the length and time scales of secondary instabilities which develop in the tilted braid between two adjacent $\mathcal{K H}$ billows. Since this is an important feature of sill flows as $\mathcal{K H}$ instabilities are known to cause significant vertical mixing by reinitiating three-dimensional motions, we present results for varying shallow slopes using temporal linear stability analysis.

The paper is organized as follows. In Sec. II the general approach adopted for the linear stability is described defining the velocity profiles and presenting the governing equations and describing the numerical solution method. Results for the effect of the bottom slope on the stability characteristics are presented and discussed in Sec. III including results for the additional effect of changing the density scale $R$. Section IV summarizes the results and includes concluding remarks.

\section{DEFINITIONS}

The stability of the interface between the two layers of a stratified system is investigated by means of linear stability analysis of the two-dimensional momentum equations, written in a coordinate system in which the longitudinal direction

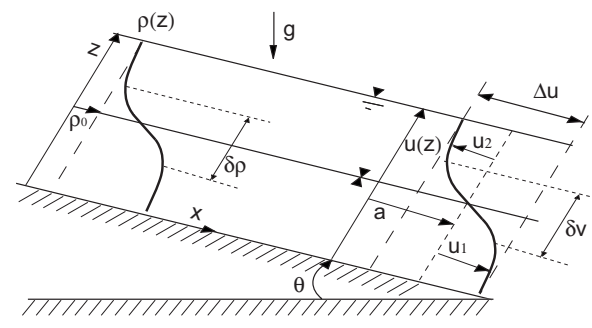

FIG. 2. Definition sketch for deriving the governing stability equation with hyperbolic tangent velocity and density profiles for a stratified two-layer flow with the notations and symbols used.

is parallel to a mild sloping bottom and the vertical direction is orthogonal to the bottom (see Fig. 2). With this definition of the coordinate system, the buoyancy term which normally appears only in the vertical equation of motion is split in one component parallel to the inclined bottom and one component that is orthogonal. This results in an additional source of instability due to the flow acceleration down the slope, and the stabilizing effect of buoyancy is reduced by the corresponding amount. The investigation of the effect of the spatial acceleration is approached differently as in Refs. 10, 13, and 14: here, the spatial acceleration is directly included in the governing stability equations and the onset and evolution of the perturbations are considered from a temporal frame of reference. This is more appropriate since we are interested in understanding the onset mechanisms of the instability at the interface of a two-layer stratified flow due to the acceleration down the slope rather than in investigating their spatial evolution.

\section{A. Velocity and density profiles}

We define the nondimensional velocity and density profiles as follows:

$$
u(z)=a+\tanh (z), \quad \rho(z)=\tanh (R z),
$$

where $a$ is the nondimensional (constant) mean advection, and $R=\delta_{\nu} / \delta_{\rho}$ is the ratio of the velocity shear layer thickness $\delta_{\nu}$ to the density layer thickness $\delta_{\rho}$ and changes the steepness of the density profile. The profiles are made dimensionless by use of the velocity scale $\Delta U / 2$ with $\Delta U=U_{1}-U_{2}$, the density scale $\Delta \rho / 2$ with $\Delta \rho=\rho_{1}-\rho_{2}$, and the vertical length scale $\delta_{v}$. We note that with the definitions of our profiles given in Eq. (1), the equality at the origin between the gradient and local Richardson numbers, Ri and $J$, respectively, is not satisfied. The choice of the hyperbolic tangent profiles for both the velocity and density distributions is justified by the good agreement between the hyperbolic tangent profiles and the experimental data, as demonstrated in Figs. 3(a) and 3(b). We note that a linear piecewise definition for the density profile might fit better to the experimental data. However, for an easier and more direct comparison of our results to those of previous studies we choose finally the hyperbolic tangent profile. 

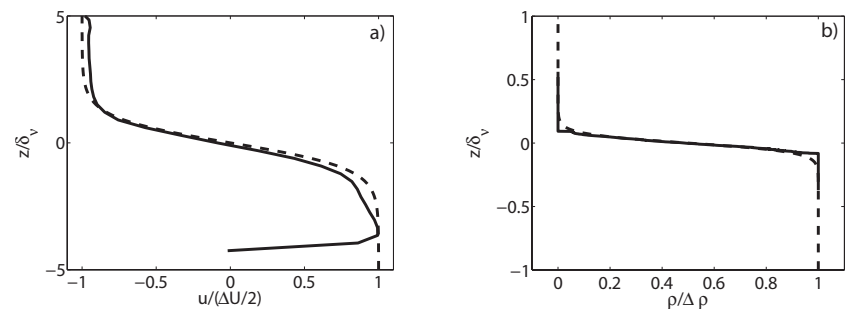

FIG. 3. Comparison between the experimental profiles (continuous line) and theoretical hyperbolic tangent (dashed line) for (a) velocity and (b) density profiles.

\section{B. Governing equations}

Even if three-dimensional effects cannot be neglected, ${ }^{16}$ we consider here a two-dimensional, unsteady, incompressible, two-layer stratified flow which can be described using the velocity components in the horizontal $x$ and vertical $z$ directions, $u$ and $w$, respectively, the density $\rho$, the pressure $p$, and a mild slope angle $\theta$ (Fig. 2).

Neglecting vertical mixing, the exact governing equations for two-dimensional flow are given by the following set of equations:

$$
\begin{aligned}
& \rho\left(u_{t}+u u_{x}+w u_{z}\right)=-p_{x}+\rho g \sin \theta+\mu\left(u_{x x}+u_{z z}\right), \\
& \rho\left(w_{t}+u w_{x}+w w_{z}\right)=-p_{z}-\rho g \cos \theta+\mu\left(w_{x x}+w_{z z}\right), \\
& \rho_{t}+u \rho_{x}+w \rho_{z}=\kappa\left(\rho_{x x}+\rho_{z z}\right), \\
& u_{x}+w_{z}=0
\end{aligned}
$$

where $g, \mu, \kappa$, and $\gamma$ are the gravitational acceleration, the molecular dynamic viscosity, the molecular diffusivity, and the coefficient of expansion for the stratifying agent, respectively, and are assumed constant. The subscripts $x$ or $z$ denotes differentiation with respect to the streamwise and vertical directions, respectively.

To perform a linear stability analysis we use the method of normal modes. To study temporally growing modes it must be assumed that the instabilities grow much faster than the flow accelerates spatially so that it can be treated as stationary. In fact, the buoyant component parallel to the bottom slope [see Eq. (2)] induces an additional acceleration in the flow direction, which is not balanced by any other force. Whereas the vertical momentum equation gives the hydrostatic balance, the buoyant acceleration in the horizontal momentum equation can only be balanced by the acceleration term $u_{t}$ given the definition of the velocity profile [Eq. (1)]. We thus define an acceleration rate $\left|u_{t} / u\right|$ by dimensional considerations of the momentum equations written for the mean state and assuming a stationary net mass flux far from the shear layer (where viscous effects can be neglected) as follows:

$$
a_{u}=\left|\frac{u_{t}}{u}\right| \sim J \sin \theta .
$$

The linear eigenproblem is obtained by adopting the standard procedure of splitting the flow field into a parallel mean component and a perturbation field, collecting terms of equal magnitude (neglecting higher order terms) and subtracting the background flow. We define the normal mode for the stream function, i.e., $\psi(x, z, t)=\mathfrak{R}\left[\phi(z) e^{l \alpha(x-c t)}\right]$, with $\Re$ designating the real component and $\psi$ defined so that $u=\partial \psi / \partial z$ and $w=-\partial \psi / \partial x$. The governing stability equation in nondimensional form reads finally

$$
\begin{aligned}
\left(\phi_{z z}-\right. & \left.\alpha^{2} \phi\right)-\frac{u_{z z}}{(u-c)} \phi+J \cos \theta \frac{\rho_{z}}{(u-c)} \phi \\
& +\frac{J \sin \theta}{l \alpha(u-c)^{2}}\left[\rho_{z z} \phi-\frac{\rho_{z} u_{z}}{(u-c)} \phi+\rho_{z} \phi_{z}\right]=0,
\end{aligned}
$$

where the density diffusion and the viscous term have been neglected. Since most of the natural flows have large Reynolds numbers and the shear layer is assumed to be far from the bottom boundary layer, this does not represent a significant restriction to our results. In Eq. (4), $u(z)$ and $\rho(z)$ are the velocity and density profiles, respectively, defined as in Eq. (1), $\phi$ denotes the complex amplitude of the disturbance, $\alpha=\alpha_{r}+\imath \alpha_{l}$ and $\omega=\omega_{r}+\imath \omega_{l}$ are the wavenumber $\left(\alpha_{r}\right)$, the spatial amplification rate $\left(\alpha_{l}\right)$, the frequency $\left(\omega_{r}\right)$, and the temporal amplification rate $\left(\omega_{l}\right)$ of the perturbation, $c=\omega / \alpha=c_{r}$ $+\imath c_{i}$ is the complex wave speed, and $J=2 \Delta \rho g \delta_{\nu} /\left(\rho_{0} \Delta U^{2}\right)$ is the bulk (local) Richardson number. Note that without slope $(\theta=0)$, Eq. (4) reduces to the Taylor-Goldstein equation.

\section{Numerical solution method}

Equation (4) has been solved using a high-order centered explicit finite difference method of order 2 on an irregular grid. ${ }^{17}$ The collocation points $\hat{z}_{j}=\lambda z_{j} / \sqrt{1-z_{j}^{2}}$, $z_{j}=-\cos [\pi(2 j-1)] /[2(N-2)]$ were mapped ${ }^{18}$ to an unbounded domain, with $\lambda$ controlling the density of grid points near the center of the domain and $N$ denoting the number of grid points. The computational domain was defined as an interval $-z_{\max } \leqslant z \leqslant z_{\max }$ (the hat ${ }^{\wedge}$ has been dropped for simplicity), where the value $z_{\max }=100 \delta_{\nu}$ was chosen so that the results are independent on the further increase in its value. The boundary conditions were defined using the asymptotic behavior of the perturbations at $z$ $\rightarrow \pm \infty$, namely, $\phi( \pm \infty)=\phi^{\prime}( \pm \infty)=0$. To check the validity of the numerical code, the results obtained solving the Taylor-Goldstein equation ( $\theta=0$, inviscid equation) were compared to the results of Holmboe ${ }^{6}$ and Hazel, ${ }^{7}$ where the exact solution for the stationary neutral stability curve in the $\left(\alpha_{r}, J\right)$ plane is known: $J=\alpha(1-\alpha)$. To obtain the results with a precision within the fourth decimal digit, a minimum number of 1200 grid points and a value of $\lambda=0.25$ were required. For the numerical solution procedure, ${ }^{19}$ we select a certain value of $R$ and a certain wavenumber and let the bulk Richardson number range between $0.01<J<0.51$. For every Richardson number, the corresponding eigenvalue $\omega$ is computed. This calculation is then repeated for different values of the wavenumber $0.01<\alpha_{r}<1.0$ and for five slope angles. Considering definition (3) small slope angles ranging from $10^{-2}$ to $10^{-1}$ are considered. The results in the following section are first presented for a fixed value of $R=1$. The case $R>1$ has also been investigated and results will be presented further below for $R=1.3, R=1.5$, and $R=2.0$. 

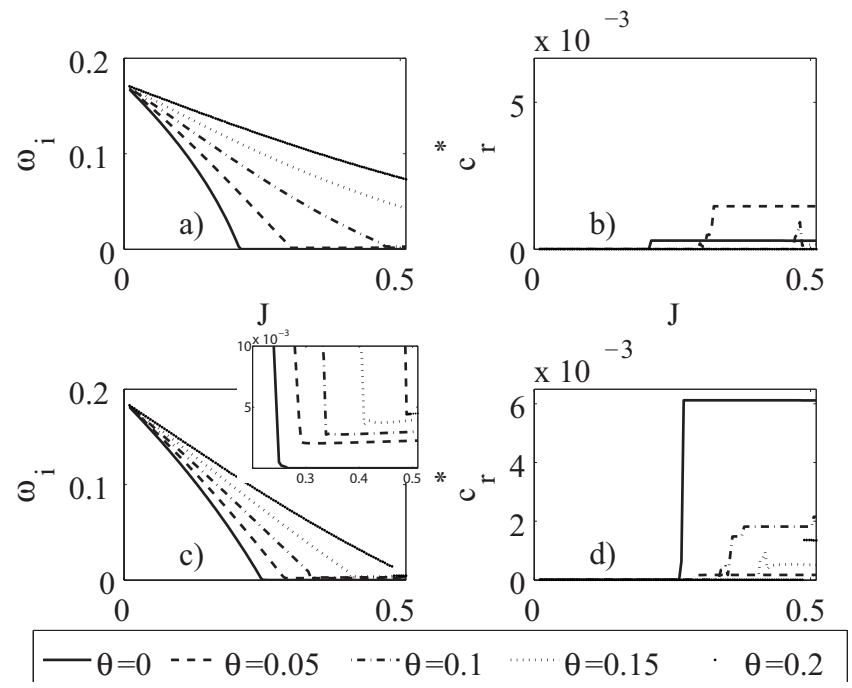

FIG. 4. Temporal amplification rates (left column) and intrinsic phase speed $c^{*}=c_{r}-a$ (right column) with respect to the Richardson number $J[$ (a) and (b)] for $\alpha_{r}=0.3$ and [(c) and (d)] for $\alpha_{r}=0.5, R=1$, and $a=0.05$. In (c) the area where the amplification rates drops to a nearly constant value are zoomed in the additional square.

\section{RESULTS AND DISCUSSION}

The mean advection $a$ in the temporal case acts as a Doppler shift in frequency ${ }^{14}$ and it affects only the real part of $\omega$ in the temporal theory. Therefore, temporal instability will be fully described by considering the intrinsic frequency of the temporal mode, $\omega_{r}^{*}=\omega_{r}-a \alpha$, as a function of $\alpha$. Moreover $\omega(\alpha)=-\bar{\omega}(-\bar{\alpha})$ (where denotes the complex conjugate) so that only positive wave numbers can be considered without any loss of generality. The corresponding intrinsic phase speed will be designated as $c_{r}^{*}$. All the results presented in the following are given for $a=0.05$.

\section{A. Results for $R=1$}

In Fig. 4 the temporal amplification rates (left column) and the intrinsic phase speed (right column) are plotted versus the Richardson number $J$ for $\alpha_{r}=0.2[(\mathrm{a})$ and (b)] and for $\alpha_{r}=0.5[(\mathrm{c})$ and (d)] for different values of the bottom slope. For a horizontal bottom $(\theta=0$, continuous line $)$ there is initially a strong decrease in the temporal amplification rate for increasing Richardson numbers. At a certain value of the Richardson number, the curve reaches a zero value. If the slope is nonzero, the temporal amplification rates do not decrease to zero in the range of Richardson numbers studied: they reach a small value and remain nearly constant. This transition occurs at higher Richardson numbers; the amplification rates decreases slower for larger slopes. Details are shown in the zoomed area in Fig. 4(c). The nearly constant value reached by the amplification rates is larger for larger values of the bottom slope.

The fact that the amplification rate does not become zero if $\theta \neq 0$ could suggest that the point at which the amplification rates become nearly constant is not the transition from unstable to stable modes but rather could represent the transition from $\mathcal{K H}$ to $\mathcal{H}$ modes, characterized with a nonzero phase speed [see Figs. 4(b) and 4(d)]. $\mathcal{H}$ waves are neutral in

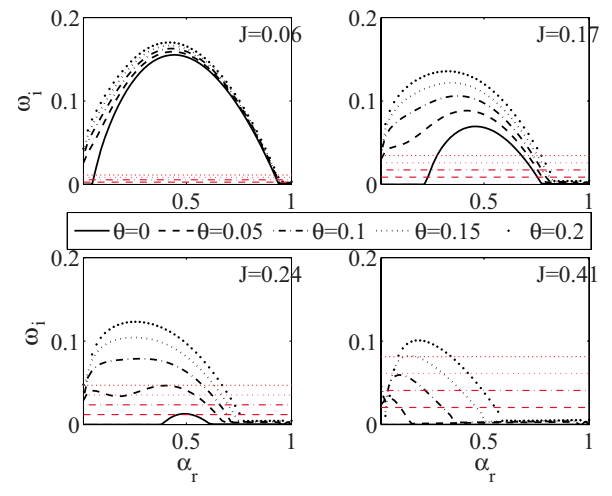

FIG. 5. (Color online) Amplification rates $\omega_{i}$ vs the wavenumber for fixed values of the Richardson number $J$. The larger amplification rates are those of $\mathcal{K H}$ instabilities, characterized by a zero phase speed. The smaller amplification rates on the right hand side of each figure being in the order of $10^{-3}$ corresponds to $\mathcal{H}$. The horizontal lines denotes the limits of the acceleration rate $a_{u}=J \sin \theta$ for the different Richardson numbers and slope angles owing the same line style as given for the different slopes. The amplification rates of the $\mathcal{H}$ modes are clearly below the limit of the flow acceleration and thus are meaningless.

the case of a zero bottom slope as the amplification rates become zero ([see Figs. 4(a) and 4(c)], continuous lines). However, as demonstrated in the zoomed area in Fig. 4(c), the amplification rates of the possible $\mathcal{H}$ modes are in the order of $10^{-3}$, and are thus much smaller than the acceleration rates defined in Eq. (3): the $\mathcal{H}$ instability does not grow fast enough as the flow accelerates spatially. This is also well shown in Fig. 5 where the amplification rates are plotted versus the wavenumber. The constant horizontal lines denote the limit for the acceleration rate $a_{u}=J \sin \theta$ for the different slope angles. The amplification rates relative to the $\mathcal{H}$ modes in the right bottom edge of each figure are well under these limit lines.

Simulations were also run for $10^{-4}<\theta<10^{-2}$ to check if $\mathcal{H}$ modes are possible for much smaller spatial acceleration rates. However, the results showed that for these ranges of slopes, no $\mathcal{H}$ modes develop. We thus conclude that only $\mathcal{K H}$ instabilities are possible for $\theta>0$.

The regions of maximal amplification rates in Fig. 5 correspond to the $\mathcal{K H}$ mode (characterized with an intrinsic zero phase speed $c_{r}^{*}$, see Fig. 4) whatever the value of the bottom slope. The fact that the curves are asymmetric with respect to $\alpha_{r}=0.5$ for larger Richardson numbers indicates that long waves are more unstable while shorter waves becomes more and more indifferent. This is also shown in Fig. 6 , which shows continuously decreasing values for the maximal amplification rates (a) and the corresponding wavenumber (b), plotted versus the Richardson numbers. The decreasing rate is lower for larger slope angles.

The transition boundary, found where $c_{r}^{*}$ becomes nonzero, is plotted in Fig. 7 for the different slopes, taking into account also the limit set by the spatial acceleration rate. The results for $\theta=0$ are in good agreement with previous results (continuous line). If the slope angle is nonzero, the unstable regions where $\mathcal{K H}$ modes exist are larger particularly for smaller wavenumbers. These results suggest that increasing the bottom slope has a similar influence on the stability of 

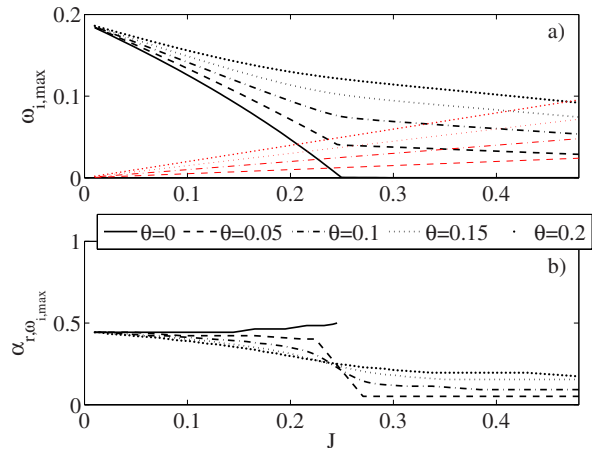

FIG. 6. (Color online) (a) Maximal amplification rate and (b) corresponding wavenumber for different Richardson numbers. The maximal amplification rates are found for the $\mathcal{K} \mathcal{H}$ modes.

the flow as increasing the parameter $R$ for the density profile with nonzero phase speed (see Fig. 8 in Ref. 7). In fact, from a physical point of view, we expect that both a bottom slope and an increased ratio $R$ have the effect of destabilizing the flow, as both terms modify the contribution of the buoyancy term in the stability equation (4).

In Fig. 8 the transition boundaries for the four slopes are plotted along with the curve of maximal amplification rates. Herein, it is shown that the wavenumbers corresponding to the maximal amplification rates $\alpha_{r, \omega_{i, \max }}$ decrease as the Richardson number increases.

This can be seen by better reconsidering Fig. 6(b): while the most amplified wavenumber $\alpha_{r, \max }$ is slightly increasing for the horizontal flow reaching the well known value of 0.5 , at $J=0.25$, the curves for $\theta=0$ decrease and present an inflection point at $J=0.25$. For a fixed value of $J<0.25$, the value of $\alpha_{r, \max }$ decreases if the slope is larger, while for $J>0.25$ the behavior is opposite. At $J=0.25$, for all slopes these values of the wavenumber collapse at the same value of $\alpha_{r}=0.25$. At this point also the curves of the amplification rates for $\theta>0$ change and their slopes become smaller [see Fig. 6(a)].

\section{B. Additional effect of changing the density scale $(R \neq 1)$}

Figure 9(a) shows experimental values of the parameter $R$ for an experiment of a two-layer equal exchange flow down a mild slope $\left(\theta=0.2 \mathrm{rad}, \Delta \rho / \rho_{0}=0.3 \%, J \sim 0.25\right)$ as a function of the normalized longitudinal direction (with $L$ being the length of the slope). The values range from 1 to 1.8 and are under the critical value for the formation of $\mathcal{H}$ instabilities of 2.0 predicted by Alexakis. ${ }^{8}$ Based on this experimental evidence, the effect of changing the density scale $R$ will be investigated in the following under consideration of three cases: $R=1.33, R=1.5$, and $R=2.0$.

By our definition of the velocity and density profiles, the local and gradient Richardson numbers, $J$ and $\mathrm{Ri}$, respectively, are not the same at the origin if $R \neq 1$. The ratio between the gradient and the local Richardson number $\operatorname{Ri}(z) / J=R \cosh ^{4}(z) / \cosh ^{2}(R z)$ as a function of the vertical coordinate $z$ is plotted in Fig. 9(b) for different values of the parameter $R$. As it was noticed by Hazel, ${ }^{7}$ it is worthwhile to note that for $R<\sqrt{2}$ the ratio monotonically increases over

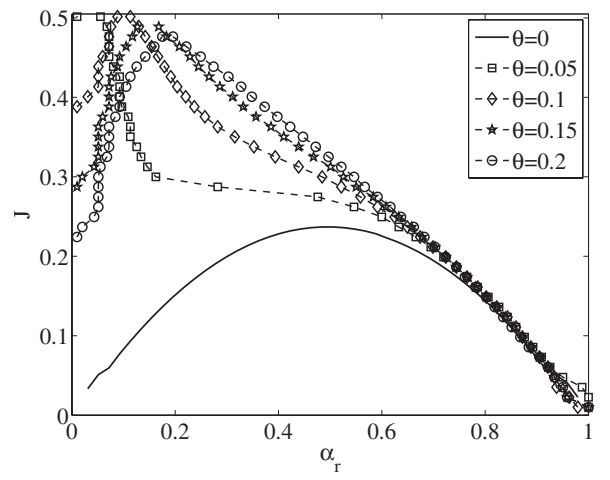

FIG. 7. Stability boundaries for different slope angles, $R=1$, and $a=0.05$. The unstable regions are increased for larger slope angles.

the depth. For $\sqrt{2}<R<2$ the ratio first decreases, reaches a minimum, and then increases again, while for $R=2$ it decreases over all the vertical domain. This behavior will be further discussed below.

Figure 10 shows the stability boundaries for the different slopes and values of the density scale $R$, taking into account the limit set by the spatial acceleration rate $a_{u}$. For $\theta=0$ the unstable regions are reduced as a result of our scaling choice for the density and velocity profiles. Compared to the case $R=1$, for $R=1.3$ and $R=1.5$ and $\theta \neq 0$, stable regions are extended to smaller wavenumbers if $J<0.3$. For $R=2$ and $\theta \neq 0, \mathcal{K H}$ modes appear again for larger $J$ and small wavenumbers. This suggests that for very sharp density profiles, i.e., approaching the step profile, $\mathcal{K H}$ instabilities occur for smaller Richardson numbers where there is a strong velocity shear and they have large wavelengths. Smaller wavelengths, on the other hand, become rapidly stable.

Figure 11 shows the maximum amplification rates and the corresponding wavenumber versus the local Richardson number for the different slope angles and the value of the density scale parameter $R$. Compared to the case $R=1$ (see Fig. 6) the maximal amplification rates do not decrease
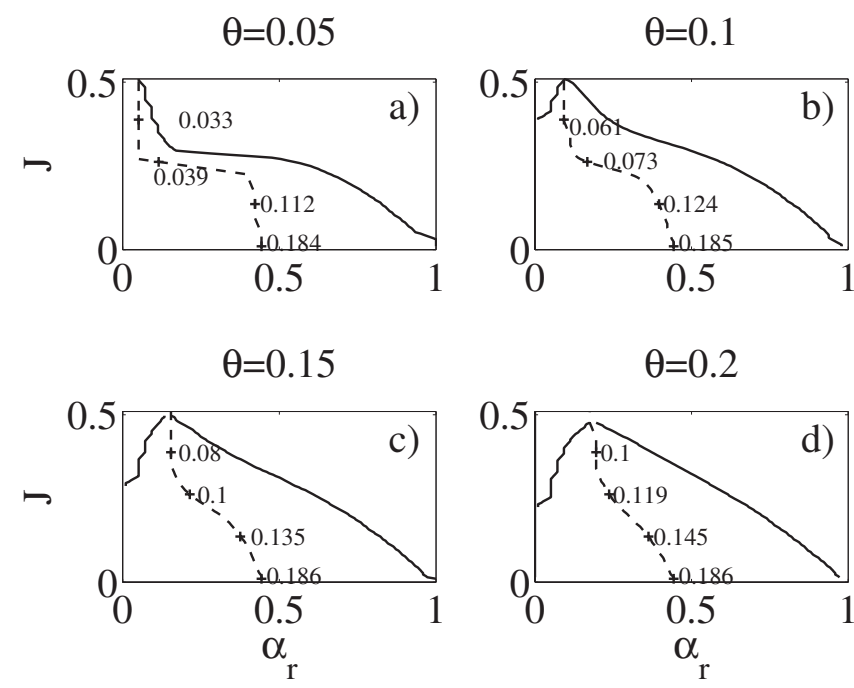

FIG. 8. Stability boundaries for $R=1$ and $a=0.05$ with the relative line of maximal amplification rates $\omega_{l, \max }$, for (a) $\theta=0.05$, (b) $\theta=0.1$, (c) $\theta=0.15$, and (d) $\theta=0.2$. 

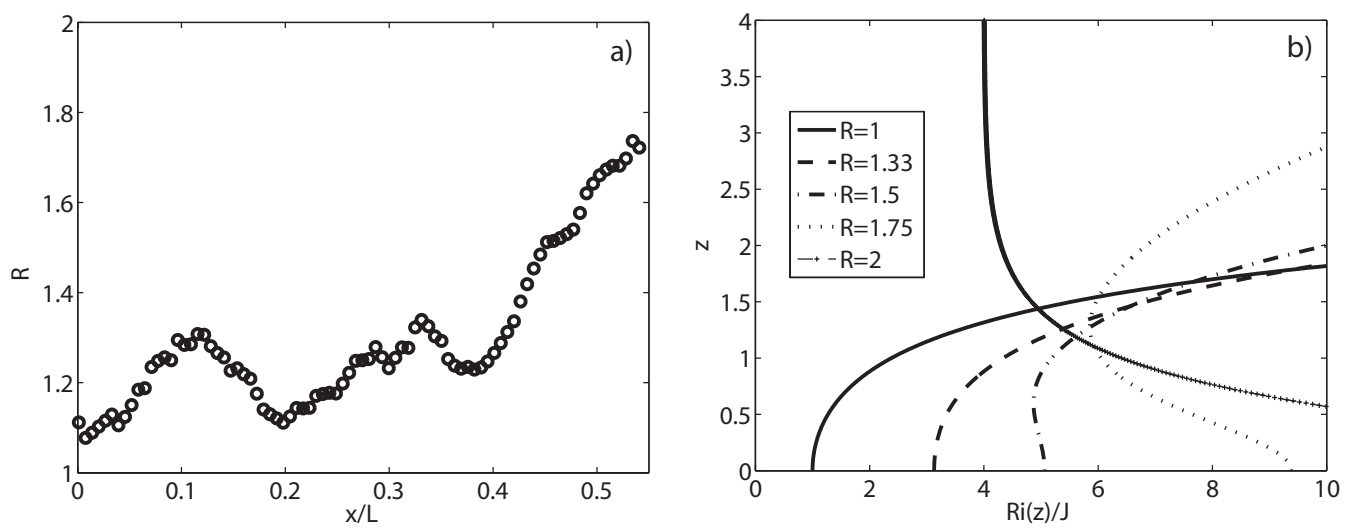

FIG. 9. (a) Experimental values for the density scale parameter $R=\delta_{\nu} / \delta_{\rho}$ down a slope in the longitudinal direction, showing values ranging from 1 to 2 . (b) Behavior of the ratio between the gradient and the local Richardson number $\operatorname{Ri}(z) / J=R \cosh ^{4}(z) / \cosh ^{2}(R z)$ in the vertical direction $z$ for different values of the parameter $R$.

monotonically as the local Richardson number increases, but at a certain value they reach a minimum and then start to grow again. This value of the Richardson number $J$ is larger for larger slope angles and its value also increases as the density scale $R$ decreases. As the slope increases, also the growing rate after reaching the minimum increases. This means that in the presence of a bottom slope and if the density scale differs from the velocity length scale, the stability of the flow cannot be completely described by looking at the behavior of the Richardson number. For the case $R=1$, the maximal amplification rates decreases monotonically whatever the value of the Richardson number $J$, and the Richardson number is an indicator for stability: larger Richardson numbers indicate more stable flows, as buoyancy prevails over the destabilizing effect of the velocity shear. As the bottom slope increases, this effect is reduced as part of the buoyancy works for the destabilization due to the acceleration down the slope and this explains the more gentle decreasing rates of the amplification rates versus the Richardson numbers as demonstrated from the results in Fig. 6 for $\theta=0$. However, if the density scale changes, the rule of the Richardson number applies only under a certain value of the Richardson number: after this threshold, the destabilizing part of buoyancy due to the spatial acceleration overwhelms the stabilizing effect of the buoyancy. This also explains why the growing rate for the amplification rate increases for larger slope angles and why it occurs at smaller Richardson

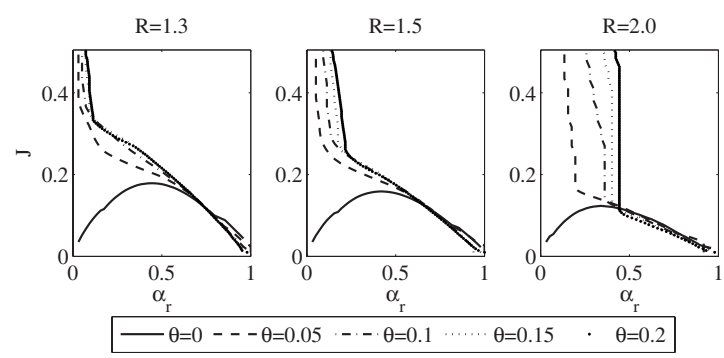

FIG. 10. Transitions boundaries from $\mathcal{K H}$ to stable flow for different slope angles, $R=1.33, R=1.5$, and $R=2.0$ and $a=0.05$ (inviscid solution). The instability regions are increased for larger slope angles. Transition to stable flow occur at lower Richardson numbers and wavenumbers as the density scale parameter $R$ increases. numbers $J$. One effect of changing the density scale is thus to amplify the destabilizing effect originated by the velocity shear: larger values of $R$ result on one side in more stable interfaces due to a larger density gradient, but on the other side, the additional buoyant acceleration in the flow direction is also larger. The interplay between the two related scales of density and velocity shear is dominated at a first instance by the density variations, with a dominant stabilizing effect. Then, both the velocity shear and the destabilizing contribution of buoyancy cause unstable interfaces.

The curves relative to the wavenumbers $\alpha_{r, \omega_{i, \max }}$ corresponding to the maximal amplification rates $\omega_{i, \max }$ are shown in Fig. 11. At a certain value of $J$ the wavenumber $\alpha_{r, \omega_{i, \max }}$ stops decreasing and becomes constant. These constant values increases as the density scale $R$ increases, especially for small slopes. This demonstrates that one of its effect is to make the flow unstable for shorter waves. Also for $R=2$ there is only one intersection point and it occurs at smaller Richardson numbers.

It is also interesting to observe the variation in the maximum amplification rate at a given Richardson number versus the parameter $R$ (see Fig. 12): for $J=0.05$ the maximum amplification rate decreases as $R$ increases for any slope angle, even if slight decreasing rates are observed for larger slope angles. For $J=0.25$, this is only true for small slope angles; for $\theta=0.15$ and $\theta=0.2$ it decreases until $R=1.5$ [we argue that probably the minimum is reached at the critical value of $R=\sqrt{2}$, see Fig. 9(b) $]$ and then it starts to increase again. For $J=0.4$, the maximal amplification rates increase with $R$ whatever the value of the slope angle. The same behavior is observed for the corresponding wavenumber. This suggests that the change in the density scale becomes effective in destabilizing the flow only if a certain value of the Richardson number is reached, at which the destabilizing contribution of buoyancy due to the bottom inclination becomes dominant as compared to its stabilizing component.

A similar behavior is also observed in the $\left(\omega_{i}, \alpha_{r}\right)$ plane for different values of $R$ (see Fig. 13). First we look at the behavior for varying Richardson numbers $J$ fixing the value of $R$. For $R<\sqrt{2}$ (i.e., $R=1.3$ ) the most amplified region corresponding to the $\mathcal{K H}$ mode decreases and shifts to the 

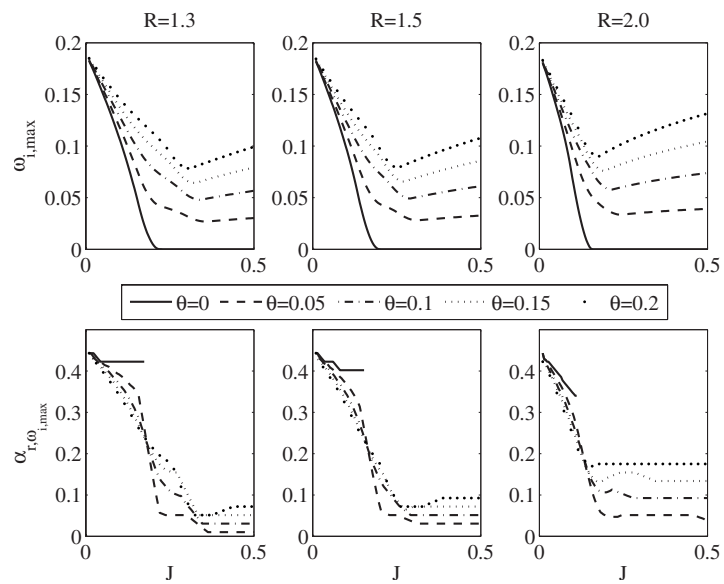

FIG. 11. Maximal amplification rates and corresponding wavenumber vs the Richardson number $J$ for different values of the density scale parameter $R$. The maximal amplification rates are found only for the $\mathcal{K} \mathcal{H}$ modes.

left to smaller wavenumbers (corresponding to longer waves) as the Richardson number increases. If $R>\sqrt{2}$, i.e., for $R=1.5$ and $R=2.0$, the most amplified region first reduces and shifts to smaller wavenumbers as $J$ increases; then the most unstable regions increase again as $J$ increases.

Fixing the value of the Richardson number $J$ and looking at the development for growing values of $R$ we note that for $J=0.1$ the most amplified regions decrease as $R$ increases. For $J=0.25$ the most amplified regions first decrease for $R=1.5$ and then they increase again for $R=2.0$. For $J=0.4$ the most amplified regions increases with growing $R$.

Based on these ascertainments, we argue that there are thresholds for both the Richardson number $J$ and for the density scale $R$ under the action of a spatial acceleration at which the stability properties change behavior: this threshold seem to be $\sqrt{2}$ for the parameter $R$ whatever the value of the slope angle, while the threshold value of the Richardson number depends on the bottom slope angle. The smaller values of the amplification rates and the corresponding range of wavenumbers are found in the transition regions for the threshold values for $J$ and $R$.

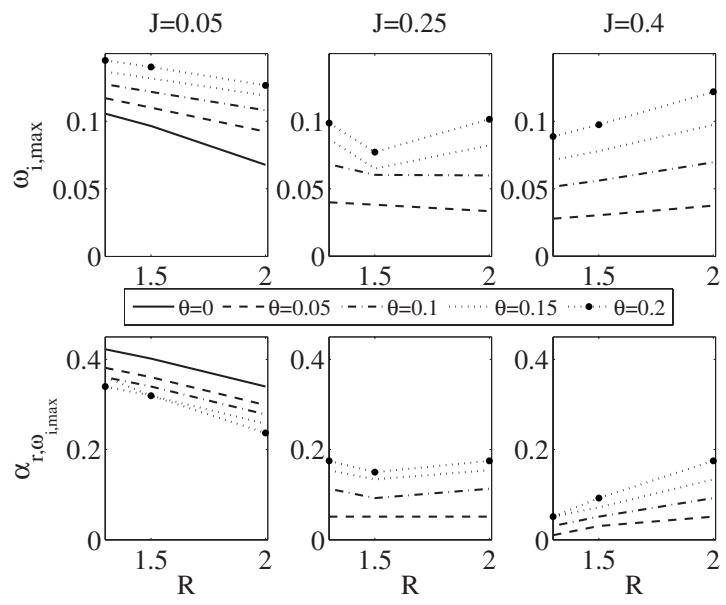

FIG. 12. Maximal amplification rates and corresponding wavenumber vs the density scale parameter $R$ for fixed values of the Richardson number. The maximal amplification rates are found for the $\mathcal{K} \mathcal{H}$ modes.
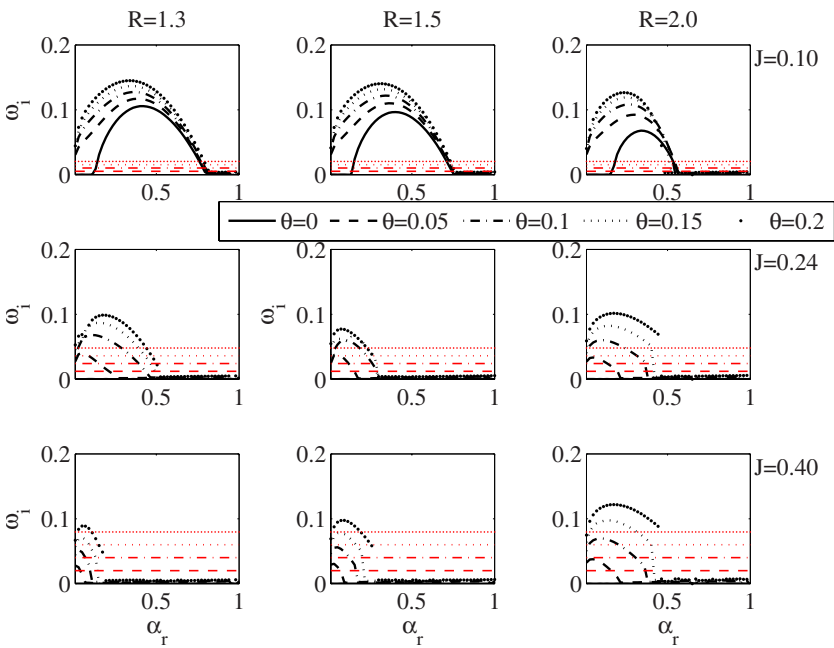

FIG. 13. (Color online) Amplification rates $\omega_{i}$ vs the wavenumber for fixed values of the Richardson number $J$ and for different values of the density scale parameter $R$. The larger amplification rates are those of $\mathcal{K H}$ instabilities.

\section{SUMMARY AND CONCLUSIONS}

A temporal linear stability analysis of a two-layer stratified exchange flow under the action of a weak mean advection has been performed in order to investigate the effect of a spatial acceleration due to an inclination of the bottom on the stability of the interface between the two layers. Conversely to previous studies, in this study the spatial acceleration is directly included in the governing stability equations instead of keeping the classical Taylor-Goldstein equation and studying the stability from a spatial frame of reference. This approach also allows a more direct analysis and comprehension of the effect of the bottom inclination on the stability properties of the flow. A modified form of the TaylorGoldstein equation has been derived, in which the buoyant term is decomposed in one (classic) component orthogonal to the inclined bottom and in one component which is parallel. The latter adds an additional acceleration in flow direction and makes the flow nonstationary, thus introducing in the physical system one additional destabilizing component near that coming from the velocity shear. To study temporally growing modes a spatial acceleration rate has been defined to set a validity limit for the calculated growth rates.

For $\theta>0$ two types of instabilities were found, namely, the $\mathcal{K H}$ instability and the $\mathcal{H}$ instability. However, the amplification rates of the $\mathcal{H}$ modes where found to be much smaller than the spatial acceleration rate. Simulations were also run for $10^{-4}<\theta<10^{-2}$, but in this slope range $\mathcal{H}$ amplification rates where found to be zero. This demonstrates that for $\theta \neq 0$ only $\mathcal{K H}$ modes are possible. The unstable regions were found to be increased for $\theta>0$, particularly for small wavenumbers. This is consistent with the fact that both a change in the density scale $R$ and an inclined bottom slope causes the flow to be destabilized. Larger bottom slopes shift the stability boundaries at larger wavenumbers. The maximal amplification rates relative to $\mathcal{K H}$ modes decrease monotonically with increasing Richardson numbers.

The additional effect of changing the density scale 
$R=\delta_{\rho} / \delta_{\nu}$ has also been analyzed. The regions characterized with $\mathcal{K} \mathcal{H}$ modes are reduced and the transition to stable flow occurs at lower Richardson numbers and wavenumbers for $R>1$. The maximal amplification rates decrease until a certain value of the Richardson number (this value is smaller for larger bottom slope angles) where they reach a minimum, then they grow again. Also converse behaviors of the characteristics of the amplification rates and the corresponding wavenumbers are observed depending if the value of the density scale $R$ exceeds the critical value of $\sqrt{2}$. When $R<\sqrt{2}$, larger Richardson numbers result in more stable interfaces with decreasing amplification rates. When $R>\sqrt{2}$ this happens until a certain value of the Richardson number, then the behavior becomes opposite. This threshold value of the Richardson number depends on the values of the slope angles and decreases for larger slopes. These results suggest that the change in the density scale becomes effective in destabilizing the flow only if a certain value of the Richardson number is reached, at which the destabilizing contribution of buoyancy due to the bottom inclination becomes dominant as compared to the stabilizing component of buoyancy. Also, the results demonstrate that the Richardson number is not the only parameter playing a key role in predicting the stability of the flow: this depends on the interplay between the different contributions of stabilizing/destabilizing forces included in other parameters such as the bottom slope $\theta$ and the density scale $R$.

\section{ACKNOWLEDGMENTS}

This research was funded by the German Research Foundation (DFG Grant No. Ji 18/12-1).
${ }^{1}$ D. M. Farmer and L. Armi, "The establishment of stratified flows over topography by small scale entrainment and mixing," Proc. R. Soc. London, Ser. A 455, 3221 (1999).

${ }^{2}$ M. Arita and G. H. Jirka, "Two layer model of saline wedge. I. Entrainment and interfacial friction," J. Hydraul. Eng. 113, 1229 (1987).

${ }^{3}$ G. A. Lawrence, R. Pieters, L. Zaremba, T. Tedford, L. Gu, S. Greco, and P. Hamblin, "Summer exchange between Hamilton Harbor and Lake Ontario," Deep-Sea Res., Part II 51, 475 (2004).

${ }^{4}$ Y. D. Afanasyev and W. R. Peltier, "On breaking internal waves over the sill in Knight Inlet," Proc. R. Soc. London, Ser. A 457, 2799 (2001).

${ }^{5}$ J. S. Turner, "The influence of molecular diffusivity on turbulent entrainment across a density interface," J. Fluid Mech. 33, 639 (1968).

${ }^{6} \mathrm{~J}$. Holmboe, "On the behavior of symmetric waves in stratified shear layers," Geophysiske Publikasjoner 24, 67 (1962).

${ }^{7}$ P. Hazel, "Numerical studies of the stability of inviscid stratified flows," J. Fluid Mech. 6, 39 (1972).

${ }^{8}$ A. Alexakis, "Marginally unstable Holmboe modes," Phys. Fluids 19, 054105 (2007).

${ }^{9}$ W. D. Smyth, G. P. Klaassen, and W. R. Peltier, "Finite amplitude Holmboe waves," Geophys. Astrophys. Fluid Dyn. 43, 181 (1988).

${ }^{10}$ A. Y. Gelfgat and E. Kit, "Spatial versus temporal instabilities in a mixing layer," J. Fluid Mech. 552, 189 (2006).

${ }^{11}$ J. R. Carpenter, G. A. Lawrence, and W. D. Smyth, "Evolution and mixing of asymmetric Holmboe instabilities," J. Fluid Mech. 582, 103 (2007).

${ }^{12}$ M. E. Negretti, D. Z. Zhu, and G. H. Jirka, "Barotropically induced interfacial waves in two-layer exchange flows over a sill," J. Fluid Mech. 592, 135 (2007).

${ }^{13}$ G. Pawlak and L. Armi, "Vortex dynamics in a spatially accelerating shear layer," J. Fluid Mech. 376, 1 (1998).

${ }^{14}$ S. Ortiz, J. M. Chomaz, and T. Loiseleux, "Spatial Holmboe instability," Phys. Fluids 14, 2585 (2002).

${ }^{15}$ W. D. Smyth, "Secondary Kelvin-Helmholtz instability in weakly stratified shear flow," J. Fluid Mech. 497, 67 (2003).

${ }^{16}$ W. D. Smyth and W. R. Peltier, "On the nonlinear evolution of columnar vortices in a rotating environment," Geophys. Astrophys. Fluid Dyn. 52, 249 (1990)

${ }^{17}$ B. Fornberg, "Calculation of weights in finite difference formulas," SIAM Rev. 40, 685 (1998).

${ }^{18}$ J. P. Boyd, Lecture Notes in Engineering 49: Chebyshev and Fourier Spectral Methods (Springer, Heidelberg, Germany, 1989).

${ }^{19}$ S. A. Socolofsky and G. H. Jirka, "Large-scale flow structures and stability in shallow flows," J. Environ. Eng. Sci. 3, 451 (2004). 